\title{
Treatment of HCV Infection with Direct-acting Antiviral Agents in Patients with HIV/HCV Co-infection: A Systematic Review
}

\author{
Seyed Moayed Alavian ${ }^{1,2,3}$, Fardin Dolatimehr ${ }^{1,2,3}$, Heidar Sharafi (ii) ${ }^{1,2,3}$, Mahdi Safi-Abadi ${ }^{1,2,3}$, \\ Mohammad Saeid Rezaee-Zavareh ${ }^{1,2,3}$, Mohammad Ehsan Bayatpour ${ }^{1,2,3}$, Hamidreza Karimi-Sari ${ }^{1,2,3}$ \\ and Saman Mohazzab-Torabi ${ }^{2,3,{ }^{*}}$ \\ ${ }^{1}$ Baqiyatallah Research Center for Gastroenterology and Liver Diseases, Baqiyatallah University of Medical Sciences, Tehran, Iran \\ ${ }^{2}$ Middle East Liver Diseases Center, Tehran, Iran \\ ${ }^{3}$ Meta-Analysis Study Group, Iran Hepatitis Network, Tehran, Iran \\ "Corresponding author: Middle East Liver Diseases Center, Tehran, Iran. Tel: +98-2188945186, Fax: +98-2188945188, Email: smntrb@gmail.com
}

Received 2018 August 05; Revised 2018 October 12; Accepted 2018 December 02.

\begin{abstract}
Context: Hepatitis C virus (HCV) infection is a significant cause of chronic liver disease in patients with human immunodeficiency virus (HIV) infection. Introduction of HCV direct-acting antiviral agents (DAAs) revolutionized the treatment of hepatitis C in patients with HIV/HCV co-infection. In this study, we systematically reviewed the treatment of chronic HCV infection in patients with HIV/HCV co-infection.

Evidence Acquisition: In this systematic review, electronic databases including PubMed, Scopus, ScienceDirect, and Web of Science were comprehensively searched using appropriate strategies containing all related keywords of "HCV", "HIV" and "DAA". Studies assessed the effectiveness of interferon-free HCV antiviral therapies in patients with HIV/HCV co-infection were evaluated for inclusion in the systematic review.

Results: After the screening of 728 records, we included 33 articles in our study, and seven different HCV antiviral regimens were evaluated. Ten studies for sofosbuvir plus simeprevir(SVR ranged from 72.2\% to 100\%), eight studies for sofosbuvir plus ribavirin (SVR ranged from 51.6\% to 91.6\%), 12 studies for sofosbuvir/ledipasvir (SVR ranged from $88.8 \%$ to 100\%), eight studies for sofosbuvir plus daclatasvir (SVR ranged between 84.6\% and 100\%), two studies for grazoprevir/elbasvir (SVR ranged from 86.6\% to 96.5\%), six studies for ombitasvir/paritaprevir/ritonavir plus dasabuvir(SVR ranged from 90.6\% to 100\%), and just one study for sofosbuvir/velpatasvir with $95.2 \%$ SVR rate.
\end{abstract}

Conclusions: This study found that treatment of HCV infection with DAAs can result in high SVR rate in patients with HIV/HCV co-infection.

Keywords: Hepatitis C, Therapy, Human Immunodeficiency Virus, Direct-Acting Antiviral Agents, Systematic Review

\section{Context}

Co-infection with human immunodeficiency virus (HIV) and hepatitis C virus (HCV) is known as a significant threat to public health $(1,2)$. Worldwide, approximately 71 million people have chronic HCV infection that 2.3 to 5 million individuals have HIV/HCV co-infection (3-5). The national survey in Iran has estimated HCV infection affects $0.3 \%$ of the overall population which $11 \%$ of them have HIV/HCV co-infection (6). Besides, HIV/HCV co-infection accelerates liver fibrosis, cirrhosis, hepatocellular carcinoma (HCC) and leads to a higher rate of liver decompensation (7-9).

In the era of various and effective treatments for HCV, although the treatment-induced clearance of HCV im- proves survival of patients with HIV/HCV co-infection, a considerable number of them continue to exist untreated due to the problem with access to HCV treatments (1012). Meanwhile, comorbid conditions like psychiatric problems, substance abuse, and drugs interactions can reduce the uptake of HCV antiviral therapy among patients with HIV/HCV co-infection (13).

In the past decades, although adding ribavirin (RBV) to pegylated-IFN lead to improvement in efficiency of patients' treatment, dissatisfied results were described in patients with HIV/HCV co-infection $(14,15)$. The new generation of DAAs caused improvement in the effectiveness of antiviral therapies and reduced drugs complications in HIV/HCV co-infected patients without consideration of liver fibrosis or treatment history (16-19). Accordingly, 
HIV guidelines have recommended anti-retroviral therapy (ART) for patients with HIV/HCV co-infection simultaneously with the HCV DAAs as supplementation $(11,20)$.

\section{Objectives}

The primary objective of this systematic review was determination of the effectiveness of HCV IFN-free DAA-containing regimens in patients with HIV/HCV coinfection.

\section{Evidence Acquisition}

\subsection{Data Resources and Search Strategies}

All steps of this systematic review were based on the PRISMA guideline for reporting systematic review (21). According to the subject of this study, we selected some appropriate keywords to be searched in the different databases. They were "simeprevir", "grazoprevir", "paritaprevir”, “daclatasvir”, “asunaprevir”, "ledipasvir”, "elbasvir”, “sofosbuvir”, “ombitasvir”, “dasabuvir”, "velpatasvir", "direct-acting antiviral”, "HIV”, "human immunodeficiency virus" AND "acquired immunodeficiency syndrome". Using these keywords, we created a specific search strategy for different databases including PubMed, Scopus, ScienceDirect, and Web of Science. Our last search was performed on 5 May, 2017. Furthermore, we used a different combination of these keywords in Google Scholar to check if we missed any related articles. This approach also helped us to evaluate the sensitivity of our search formulas. For this purpose, we screened titles of articles in Google scholar until we found 20 unrelated serial titles. After all, we checked the references of all finally included studies in our project to find any possible missed articles.

\subsection{Eligibility Criteria}

We included all studies investigating the effectiveness of HCV IFN-free treatments with DAAs (sofosbuvir, daclatasvir, ledipasvir, ombitasvir, paritaprevir, asunaprevir, dasabuvir, grazoprevir, elbasvir, velpatasvir or simeprevir) among HIV/HCV co-infected patients. Treatment effectiveness was determined using the sustained virologic response (SVR) as having undetectable HCV RNA, 12 weeks after the end of treatment. We decided to consider only cohort or clinical trial studies published in peer-reviewed journals, and in the English language for inclusion in our study. Those studies which evaluated the effectiveness of regimens containing telaprevir and boceprevir or studies with a sample size of fewer than five patients were excluded from our systematic review.

\subsection{Study Selection, Data Extraction, and Presentation}

All articles through searching databases were imported to the EndNote Software. Two investigators (SMT and FD) independently screened titles, abstracts, and fulltexts of articles, respectively and according to the selection criteria. Investigators resolved any disagreement by mutual discussion or consultation with other authors (HSH and SMA).

The same two investigators extracted the following data from each study or each appropriate treatment protocol of study: Name of the first author, publication year, sample size, HCV genotype, treatment protocol including type and number of DAAs or using RBV, duration of the treatment protocol, and the rate of SVR. For evaluating each included clinical trial, Cochrane's assessment tool was used (22). This assessment tool contains 12 items that overall score $\geq 6$ was considered as low risk for each study. For assessing the included non-randomized studies, the Newcastle-Ottawa scale (NOS) was used (23).

Included studies had a wide variation in their methodology, and we could not run meta-analysis. Therefore, we just decided to gather their relevant information and present them in a table which tries to put together different studies with the same treatment regimens.

\section{Results}

A total of 681 papers were found through database searching after removing duplications. In title screening, 533 irrelevant titles and in abstract screening, 83 irrelevant abstracts were excluded. Then, 65 full-text articles were assessed for eligibility and finally, 33 articles included in our study (Figure 1).

Finally, in this study, seven different regimens were evaluated. Ten studies assessed sofosbuvir (SOF) plus simeprevir (SMV) regimen that the lowest SVR was $72.7 \%$ and the highest was $100 \%$. Nine studies evaluated SOF plus RBV regimen, and SVR ranged between 51.6\% and 91.6\%. In the assessment of sofosbuvir/ledipasvir (SOF/LDV) combination, 12 studies were examined that the SVR rate was from $88.8 \%$ to $100 \%$. Eight studies evaluated SOF plus daclatasvir (DCV) regimen, and SVR ranged between $84.6 \%$ and 100\%. Grazoprevir/elbasvir (GZR/EBR) combination was the fifth regimen included in our study. In the assessment of this regimen, two studies were evaluated in which the lowest SVR was $86.6 \%$, and the highest SVR was $96.5 \%$. In the investigation of ombitasvir/paritaprevir/ritonavir (OBV/PTV-r) plus dasabuvir (DSV) combination, six studies were assessed. The maximum and minimum SVR rate was $100 \%$ and $90.6 \%$, respectively. The seventh regimen was the combination of sofosbuvir/velpatasvir (SOF/VEL) in which 


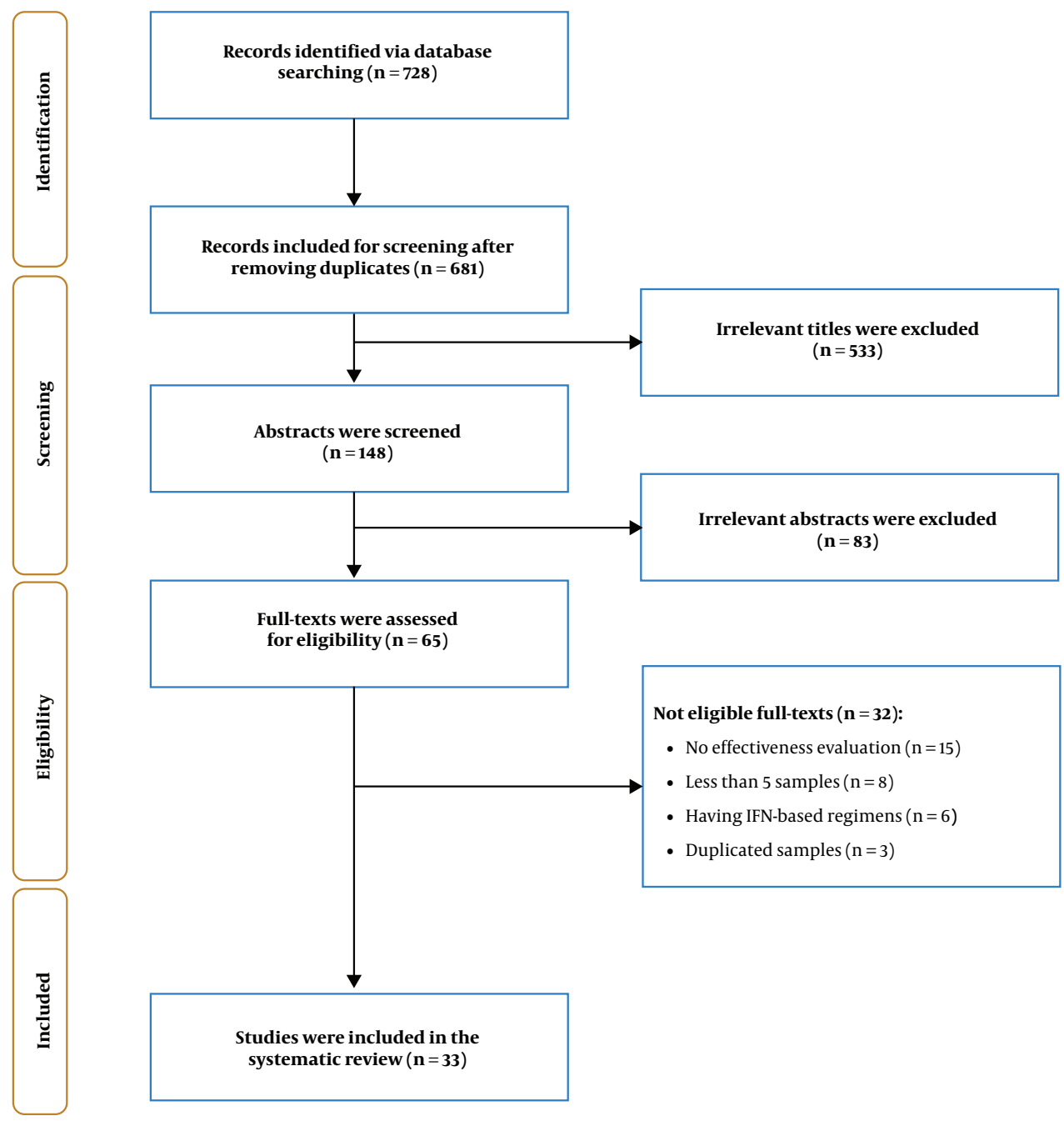

Figure 1. Screening of articles based on the PRISMA statement

only one study was evaluated, and the SVR was 95.2\%. Table 1 shows the important characteristics of the included studies and also the response rate to each HCV antiviral regimen.

\section{Discussion}

\subsection{Sofosbuvir Plus Simeprevir}

Although no dose adjustment is required in patients with renal impairment, SMV cannot be used in patients with decompensated cirrhosis and patients with previous episodes of decompensation. This antiviral agent is available as capsules containing $150 \mathrm{mg}$ of SMV and taken one capsule once daily with food. In patients receiving SMV, some antiretroviral agents are contraindicated, including cobicistat-based regimens, efavirenz, etravirine, nevirapine, ritonavir, and any HIV protease inhibitor, boosted or not by ritonavir. In the recent EASL guideline, SMVcontaining regimens are not recommended but based on 2016 version of this guideline, a combination of SOF plus SMV is appropriate just for genotype 4 in HCV monoinfected or HIV/HCV co-infected patients without cirrhosis or with compensated cirrhosis (52-54).

The most of studies found high response rate treating patients with SOF plus SMV. However, the study by Milazzo et al. found a suboptimal response rate which may be caused by HCV gene polymorphisms in NS3 (Q80K and $\mathrm{S} 122 \mathrm{~T}$ ) that detected in sequencing analysis (29). 


\subsection{Sofosbuvir Plus Ribavirin}

Sofosbuvir is an HCV NS5B polymerase inhibitor that suppresses HCV replication and life cycle. Sofosbuvir is available at the dose of $400 \mathrm{mg}$ (one tablet) and consumed once per day. There are no potential drug-drug interactions between SOF and antiretroviral drugs but in patients with severe renal impairment is contraindicated $(53,55)$. Combination of SOF plus RBV as an independent regimen for treatment of HCV infection was available in previous EASL guideline, but in 2018 version, this regimen is not available. However, none of these guidelines suggested this combination for patients with HIV/HCV co-infection $(53,54)$. Meanwhile, based on study assessments, this combination could not achieve acceptable SVR. The study by Del Bello et al., which was performed on patients with genotype-1 HCV, reported the lowest SVR rate (51.6\%); most patients in this study were cirrhotic with a history of interferon treatment (24). In another study by Hawkins et al., the SVR rate was nearly 57.1\%; most patients in this study were treatment experienced and suffered from cirrhosis (27). Seemingly, the SOF plus RBV combination is not effective in the treatment of patients with HCV infection. The SVR rate did not increase even after elongating the treatment duration.

\subsection{Sofosbuvir/Ledipasvir}

This regimen as a combination of $400 \mathrm{mg}$ SOF and 90 $\mathrm{mg}$ LDV in a single tablet is available. There was no difference in LDV plasma exposure (area under the curve (AUC)) between patients with severe hepatic impairment and normal ones. Based on the pharmacokinetic analysis in HCVinfected population, cirrhosis (compensated and decompensated) cannot cause a clinically relevant effect on the exposure to LDV. This regimen can be used in patients with mild or moderate renal impairment without dose adjustment, but the safety of the SOF/LDV combination has not been assessed in patients with severe renal impairment $(53,56)$. The SOF/LDV combination may be considered an effective regimen for patients with HCV mono-infection or HIV/HCV co-infection $(16,57)$.

Almost all antiretroviral agents may be given with this regimen. However, some combinations should be used with caution, with frequent renal monitoring due to an increase in tenofovir disoproxil fumarate (TDF) concentrations especially when ritonavir or cobicistat as pharmacokinetic enhancer are present in an antiretroviral regimen. These combinations include atazanavir/cobicistat, atazanavir/ritonavir, elvitegravir/cobicistat, darunavir/ritonavir, lopinavir/ritonavir, darunavir/cobicistat and all in combination with TDF/emtricitabine. Fortunately, after approval of the tenofovir alafenamide fumarate (TAF), due to lower plasma tenofovir levels, concerns about an interaction leading to increased tenofovir exposure have been diminished.

\subsection{Sofosbuvir Plus Daclatasvir}

Based on 2016 EASL guideline, this combination as a pangenotypic regimen was recommended for treatment of chronic HCV infection even in HIV co-infected and cirrhotic patients. Nevertheless, in the last version of EASL recommendations on treatment of hepatitis $C$, there is not any DCV-containing regimen. Daclatasvir is available in $30 \mathrm{mg}$ and $60 \mathrm{mg}$ tablets. Dose adjustment of DCV is not required for patients with renal and hepatic impairment. The assessment of drug interactions with antiretroviral agents demonstrated that there is no absolute contraindication between these drugs and DCV, but dose adjustment is required in some cases. A daily dose of DCV should be adjusted to $30 \mathrm{mg}$ with atazanavir/ritonavir and cobicistatcontaining antiretroviral regimens and increase to $90 \mathrm{mg}$ per day with efavirenz (an enzyme inducer). Also, using etravirine and nevirapine, both enzyme inducers, with DCV containing regimen are not recommended, and it may require a dosage adjustment, altered timing of administration or additional monitoring $(53,54)$. As shown in Table 1 the SVR rate in patients receiving SOF plus DCV is remarkable.

\subsection{Grazoprevir/Elbasvir}

GZR/EBR with or without RBV had few side effects so that less than $1 \%$ of patients treated with this regimen discontinued treatment due to adverse events. There is a contraindication for GZR/EBR in patients with moderate and severe hepatic impairment (Child-Pugh B and C), but dose adjustment is not required in patients suffering from renal impairment. Based on the EASL guideline, there are limitations on consuming GZR/EBR with antiretroviral agents. The nucleotide reverse transcriptase inhibitors can be used and no clinically significant interaction expected. This group of ARTs includes abacavir, lamivudine, tenofovir (either as TDF or as TAF), emtricitabine, rilpivirine, raltegravir, dolutegravir and maraviroc (53).

In the evaluation of this treatment regimen, only two studies were examined. It can be seen from the data in Table 1 that this regimen obtained relatively acceptable results.

\subsection{Ombitasvir/Paritaprevir/Ritonavir Plus Dasabuvir}

This regimen can be used in patients suffering mild hepatic impairment (Child-Pugh A) without a dose modification, but the combination of ritonavir-boosted PTV and OBV with or without DSV should not be used in patients 
with moderate and severe hepatic impairment (ChildPugh B and C). In HIV co-infected patients drug interactions need to be carefully considered. From protease inhibitors, only atazanavir and darunavir can be taken without ritonavir, and the rest of protease inhibitors are contraindicated. Efavirenz, etravirine, and nevirapine are contraindicated. ECG monitoring should be considered in case of prescribing rilpivirine. Due to additional boosting effect, cobicistat-containing regimens should not be used. Based on EASL treatment recommendation for chronic hepatitis C, in HCV mono-infected or HIV/HCV co-infected patients without cirrhosis or with compensated (ChildPugh A) cirrhosis, this regimen can be used only in genotype $1 \mathrm{~b}(53,58-61)$. As can be seen from Table 1 the most of studies found high response rate while treating patients with this regimen.

\subsection{Sofosbuvir/Velpatasvir}

In HIV/HCV co-infected patients, SOF/VEL can be used along with most of the antiretroviral agents, the exceptions being the inducing drugs efavirenz, etravirine, and nevirapine. VEL exposure would decrease by about 50\% if efavirenz consumed concurrently. Secondary to inhibiting P-gp by SOF/VEL exposure of tenofovir increases so renal adverse events should have been monitored in patients on a regimen containing TDF. This regimen can be used for all HCV genotypes in non-cirrhotic patients but in patients with compensated (Child-Pugh A) cirrhosis, SOF/VEL is not recommended for HCV genotype 3. SOF/VEL, with daily weight-based RBV (all genotypes) can be used in patients with decompensated (Child-Pugh B or C) cirrhosis (with no evidence of HCC) that are not on the waiting list for liver transplant and in patients waiting for liver transplantation with MELD score < 18 - 20 (53, 62-64). In the evaluation of this treatment regimen, only one study was assessed. In the study by Younossi et al., patients received $400 \mathrm{mg}$ of SOF and $100 \mathrm{mg}$ of VEL in a single tablet. The SVR rate was $95.2 \%$ in 106 patients with different HCV genotypes, who received 12 weeks of treatment (51).

\section{Conclusions}

With more than 70 million worldwide prevalence, hepatitis C is raised as a global concern (65). 20\% SVR was the result of HCV treatment with pegylated-interferon and adding RBV increased the treatment efficacy by up to 50\% $(66,67)$.

Introduction of first DAAs in 2011 was one of the most significant recent achievements in the field of hepatology and liver diseases makes hepatitis $C$ a curable disease (68, $69)$. The emergence of the new generation of DAA agents led to creating IFN-free regimens with more than $95 \%$ efficacy (70-72). Therefore, treatment of HCV patients seems to be available.

These days, treatment of special patient populations functions as a challenge to HCV treatment. Patients coinfected with HIV are one of these particular groups that need special attention because it is widespread, particularly among people who inject drug, and they exhibit lower rates of spontaneous HCV clearance. On the other hand, $\mathrm{HIV} / \mathrm{HCV}$ patients are prone to HCV-related liver diseases such as cirrhosis and HCC and historically had lower SVR rate during chronic HCV treatment than mono-infected patients (73).

As treatment of HCV improved after approval of new all-oral regimens of DAAs, the efficacy and safety of HIV/HCV co-infected patients' treatment had also been dramatically improved $(39,74)$. It is also noted in the HIV guidelines that treatment of HIV/HCV patients completed by the combination of ARTs and DAAs and only point in this area is drug interactions $(20,75)$.

The result of our systematic review demonstrated that an IFN-free regimen that contains the new generation of DAA agents could provide a high SVR rate. So identifying these patients and choosing appropriate treatment will lead to satisfactory therapeutic results. On the other hand, health policymakers should design screening programs to identify patients with HIV/HCV co-infection and make the new HCV medicines available at affordable prices.

\section{Footnotes}

Authors' Contribution: Concept: Saman MohazzabTorabi, Fardin Dolatimehr, Heidar Sharafi, Seyed Moayed Alavian; data acquisition: Saman Mohazzab-Torabi, Fardin Dolatimehr, Heidar Sharafi, Mahdi Safi-Abadi, Hamidreza Karimi-Sari, Seyed Moayed Alavian; data analysis and interpretation: Saman Mohazzab-Torabi, Fardin Dolatimehr, Heidar Sharafi, Mohammad Saeid Rezaee-Zavareh, Mohammad Ehsan Bayatpour, Hamidreza Karimi-Sari, Seyed Moayed Alavian; drafting the manuscript: Saman Mohazzab-Torabi, Fardin Dolatimehr, Heidar Sharafi, Mohammad Saeid Rezaee-Zavareh, Seyed Moayed Alavian; critical revising of the manuscript: Heidar Sharafi, Seyed Moayed Alavian; final approval of the manuscript: all authors.

Conflict of Interest: Authors declare that there is no conflict of interest.

Funding/Support: None declared. 


\section{References}

1. Platt L, Easterbrook P, Gower E, McDonald B, Sabin K, McGowan C, et al. Prevalence and burden of HCV co-infection in people living with HIV: A global systematic review and meta-analysis. Lancet Infect Dis. 2016;16(7):797-808. doi: 10.1016/S1473-3099(15)00485-5. [PubMed: 26922272].

2. Sulkowski MS, Eron JJ, Wyles D, Trinh R, Lalezari J, Wang C, et al Ombitasvir, paritaprevir co-dosed with ritonavir, dasabuvir, and ribavirin for hepatitis $\mathrm{C}$ in patients co-infected with HIV-1: A randomized trial. JAMA. 2015;313(12):1223-31. doi: 10.1001/jama.2015.1328. [PubMed: 25706092].

3. Word Haelth Organization. Global alert and response (GAR) hepatitis $C$ 2012. 2017.

4. Shepard CW, Finelli L, Alter MJ. Global epidemiology of hepatitis C virus infection. Lancet Infect Dis. 2005;5(9):558-67. doi: 10.1016/S14733099(05)70216-4. [PubMed: 16122679].

5. Staples CT Jr, Rimland D, Dudas D. Hepatitis C in the HIV (human immunodeficiency virus) Atlanta V.A. (veterans affairs medical center) cohort study (HAVACS): The effect of coinfection on survival. Clin Infect Dis. 1999;29(1):150-4. doi: 10.1086/520144. [PubMed:10433578].

6. Alavian SM, Hajarizadeh B, Ahmadzad-Asl M, Kabir A, BagheriLankarani K. Hepatitis B virus infection in Iran: A systematic review. Hepat Mon. 2008;8(4)

7. Martin-Carbonero L, Benhamou Y, Puoti M, Berenguer J, Mallolas J, Quereda C, et al. Incidence and predictors of severe liver fibrosis in human immunodeficiency virus-infected patients with chronic hepatitis C: A European collaborative study. Clin Infect Dis. 2004;38(1):12833. doi: 10.1086/380130. [PubMed: 14679458].

8. Benhamou Y, Bochet M, Di Martino V, Charlotte F, Azria F, Coutellier A, et al. Liver fibrosis progression in human immunodeficiency virus and hepatitis $C$ virus coinfected patients. The multivirc group. Hepatology.1999;30(4):1054-8. doi:10.1002/hep.510300409. [PubMed: 10498659].

9. Miro JM, Stock P, Teicher E, Duclos-Vallee JC, Terrault N, Rimola A Outcome and management of HCV/HIV coinfection pre- and postliver transplantation. A 2015 update. J Hepatol. 2015;62(3):701-11. doi: 10.1016/j.jhep.2014.10.032. [PubMed: 25450714].

10. Simmons B, Saleem J, Heath K, Cooke GS, Hill A. Long-term treatment outcomes of patients infected with hepatitis $C$ virus: A systematic review and meta-analysis of the survival benefit of achieving a sustained virological response. Clin Infect Dis. 2015;61(5):73040. doi: 10.1093/cid/civ396. [PubMed: 25987643]. [PubMed Central: PMC4530725].

11. Pawlotsky JM, Aghemo A, Back D, Dusheiko G, Forns X; European Association for Study of Liver, et al. EASL recommendations on treatment of hepatitis C 2015. J Hepatol. 2015;63(1):199-236. doi: 10.1016/j.jhep.2015.03.025. [PubMed: 25911336].

12. Reiberger T, Obermeier M, Payer BA, Baumgarten A, Weitner L, Moll A, et al. Considerable under-treatment of chronic HCV infection in HIV patients despite acceptable sustained virological response rates in a real-life setting. Antivir Ther. 2011;16(6):815-24. doi:10.3851/IMP1831. [PubMed: 21900713].

13. Patel M, Rab S, Kalapila AG, Kyle A, Okosun IS, Miller L. Highly successful hepatitis $\mathrm{C}$ virus (HCV) treatment outcomes in human immunodeficiency virus/HCV-coinfected patients at a large, urban, ryan white clinic. Open Forum Infect Dis. 2017;4(2):ofx062. doi: 10.1093/ofid/ofx062. [PubMed: 28534036]. [PubMed Central: PMC5421352].

14. Mandorfer M, Neukam K, Reiberger T, Payer BA, Rivero A, Puoti M, et al. The impact of interleukin 28B rs12979860 single nucleotide polymorphism and liver fibrosis stage on response-guided therapy in HIV/HCV-coinfected patients. AIDS. 2013;27(17):2707-14. doi: 10.1097/01.aids.0000432460.44593.ef. [PubMed: 23835502].

15. Haj-Sheykholeslami A, Keshvari M, Sharafi H, Pouryasin A, Hemmati $\mathrm{K}$, Mohammadzadehparjikolaei F. Interferon-lambda polymor- phisms and response to pegylated interferon in Iranian hepatitis C patients. World J Gastroenterol. 2015;21(29):8935-42. doi: 10.3748/wjg.v21.i29.8935. [PubMed: 26269684]. [PubMed Central: PMC4528037].

16. Naggie S, Cooper C, Saag M, Workowski K, Ruane P, Towner WJ, et al. Ledipasvir and Sofosbuvir for HCV in patients coinfected with HIV-1. NEngl J Med. 2015;373(8):705-13. doi: 10.1056/NEJMoa1501315. [PubMed: 26196665]. [PubMed Central: PMC4892372].

17. Rockstroh JK, Nelson M, Katlama C, Lalezari J, Mallolas J, Bloch M, et al. Efficacy and safety of grazoprevir (MK-5172) and elbasvir (MK8742 ) in patients with hepatitis $\mathrm{C}$ virus and HIV co-infection (CEDGE CO-INFECTION): A non-randomised, open-label trial. Lancet HIV. 2015;2(8):e319-27. doi: 10.1016/S2352-3018(15)00114-9. [PubMed: 26423374].

18. Alavian SM, Hajarizadeh B, Bagheri Lankarani K, Sharafi H, Ebrahimi Daryani N, Merat S, et al. Recommendations for the clinical management of hepatitis $C$ in Iran: A consensus-based national guideline. Hepat Mon. 2016;16(8). e40959. doi:10.5812/hepatmon.guideline. [PubMed: 27799966]. [PubMed Central: PMC5075356].

19. Alavian SM, Sharafi H. Update on recommendations for the clinical management of hepatitis C in Iran 2017. Hepat Mon. 2017;17(11). e63956. doi: 10.5812/hepatmon.63956.

20. Cope R, Pickering A, Glowa T, Faulds S, Veldkamp P, Prasad R. Majority of HIV/HCV patients need to switch antiretroviral therapy to accommodate direct acting antivirals. AIDS Patient Care STDS. 2015;29(7):37983. doi:10.1089/apc.2015.0004. [PubMed: 26066094].

21. Moher D, Liberati A, Tetzlaff J, Altman DG, Prisma Group . Preferred reporting items for systematic reviews and meta-analyses: The PRISMA statement. PLoS Med. 2009;6(7). e1000097. doi: 10.1371/journal.pmed.1000097. [PubMed:19621072]. [PubMed Central: PMC2707599].

22. Higgins JP, Altman DG. Assessing risk of bias in included studies. Cochrane handbook for systematic reviews of interventions: Cochrane book series. 2008. p. 187-241.

23. Stang A. Critical evaluation of the Newcastle-Ottawa scale for the assessment of the quality of nonrandomized studies in meta-analyses. Eur J Epidemiol. 2010;25(9):603-5. doi: 10.1007/s10654-010-9491-z. [PubMed: 20652370].

24. Del Bello D, Cha A, Sorbera M, Bichoupan K, Levine C, Doyle E, et al. Real-world sustained virologic response rates of sofosbuvircontaining regimens in patients coinfected with hepatitis $C$ and HIV. Clin Infect Dis. 2016;62(12):1497-504. doi: 10.1093/cid/ciw119. [PubMed: 26936665]. [PubMed Central: PMC4885645].

25. Johnson TM, Sison R, Fallon JP, Shukla PP, Bhattarai S, Galang $\mathrm{H}$, et al. Clinical experience with dolutegravir/abacavir/lamivudine in HIV-HCV co-infected patients treated with a sofosbuvir-based regimen-safety and efficacy. HIV Clin Trials. 2016;17(6):242-5. doi: 10.1080/15284336.2016.1248625. [PubMed: 27846791].

26. Grant JL, Hawkins C, Brooks H, Palella FJ, Koppe SW, Abecassis MM, et al. Successful sofosbuvir-based therapy in HIV/hepatitis $C$ virus coinfected liver transplant recipients with recurrent hepatitis $C$ virus infection. AIDS. 2016;30(1):93-8. doi: 10.1097/QAD.0000000000000887. [PubMed: 26731756]. [PubMed Central: PMC4754111].

27. Hawkins C, Grant J, Ammerman LR, Palella F, McLaughlin M, Green R, et al. High rates of hepatitis $\mathrm{C}$ virus (HCV) cure using direct-acting antivirals in HIV/HCV-coinfected patients: A real-world perspective. J Antimicrob Chemother. 2016;71(9):2642-5. doi: 10.1093/jac/dkw203. [PubMed: 27330060]. [PubMed Central: PMC4992854].

28. Merli M, Galli L, Marinaro L, Ariaudo A, Messina E, Uberti-Foppa C, et al. Pharmacokinetics of dolutegravir and rilpivirine in combination with simeprevir and sofosbuvir in HIV/hepatitis C virus-coinfected patients with liver cirrhosis. JAntimicrob Chemother. 2017;72(3):812-5. doi: 10.1093/jac/dkw492. [PubMed: 27999010].

29. Milazzo L, Lai A, Calvi E, Ronzi P, Micheli V, Binda F, et al. Direct-acting antivirals in hepatitis C virus (HCV)-infected and HCV/HIV-coinfected 
patients: Real-life safety and efficacy. HIV Med. 2017;18(4):284-91. doi: 10.1111/hiv.12429. [PubMed: 27477612].

30. Bruno G, Saracino A, Fabrizio C, Scudeller L, Milano E, Dell'Acqua $\mathrm{R}$, et al. Safety and effectiveness of a 12-week course of sofosbuvir and simeprevir +/- ribavirin in HCV-infected patients with or without HIV infection: A multicentre observational study. Int J Antimicrob Agents. 2017;49(3):296-301. doi: 10.1016/j.ijantimicag.2016.11.030. [PubMed: 28163136].

31. Montes ML, Olveira A, Ahumada A, Aldamiz T, Garcia-Samaniego J, Clemente A, et al. Similar effectiveness of direct-acting antiviral against hepatitis $C$ virus in patients with and without HIV infection. AIDS. 2017;31(9):1253-60. doi: 10.1097/QAD.0000000000001465. [PubMed: 28358742].

32. Piroth L, Wittkop L, Lacombe K, Rosenthal E, Gilbert C, Miailhes P, et al. Efficacy and safety of direct-acting antiviral regimens in HIV/HCVco-infected patients - French ANRS CO13 HEPAVIH cohort. J Hepatol. 2017;67(1):23-31. doi: 10.1016/j.jhep.2017.02.012. [PubMed: 28235612].

33. Campos-Varela I, Moreno A, Morbey A, Guaraldi G, Hasson H, Bhamidimarri KR, et al. Treatment of severe recurrent hepatitis $C$ after liver transplantation in HIV infected patients using sofosbuvir-based therapy. Aliment Pharmacol Ther. 2016;43(12):1319-29. doi: 10.1111/apt.13629. [PubMed: 27098374].

34. Molina JM, Orkin C, Iser DM, Zamora FX, Nelson M, Stephan C, et al. Sofosbuvir plus ribavirin for treatment of hepatitis $C$ virus in patients co-infected with HIV (PHOTON-2): A multicentre, open-label non-randomised, phase 3 study. Lancet. 2015;385(9973):1098-106. doi 10.1016/S0140-6736(14)62483-1. [PubMed: 25659285].

35. Younossi ZM, Stepanova M, Sulkowski M, Naggie S, Puoti M, Orkin $\mathrm{C}$, et al. Sofosbuvir and ribavirin for treatment of chronic hepatitis $C$ in patients coinfected with hepatitis $C$ virus and HIV: The impact on patient-reported outcomes. J Infect Dis. 2015;212(3):36777. doi: 10.1093/infdis/jiv005. [PubMed: 25583164]. [PubMed Central: PMC5007583].

36. Sulkowski MS, Naggie S, Lalezari J, Fessel WJ, Mounzer K, Shuhart $\mathrm{M}$, et al. Sofosbuvir and ribavirin for hepatitis $\mathrm{C}$ in patients with HIV coinfection. JAMA. 2014;312(4):353-61. doi: 10.1001/jama.2014.7734. [PubMed: 25038354]. [PubMed Central: PMC4997358].

37. El Sayed A, Barbati ZR, Turner SS, Foster AL, Morey T, Dieterich DT, et al. Sofosbuvir in the treatment of early HCV infection in HIV-infected men. HIV Clin Trials. 2017;18(2):60-6. doi 10.1080/15284336.2017.1280594. [PubMed: 28183221].

38. Cooper C, Naggie S, Saag M, Yang JC, Stamm LM, Dvory-Sobol H, et al. Successful re-treatment of hepatitis $C$ virus in patients coinfected with HIV who relapsed after 12 weeks of ledipasvir/sofosbuvir. Clin Infect Dis. 2016;63(4):528-31. doi: 10.1093/cid/ciw349. [PubMed: 27225242]. [PubMed Central: PMC4967607].

39. Osinusi A, Townsend K, Kohli A, Nelson A, Seamon C, Meissner $\mathrm{EG}$, et al. Virologic response following combined ledipasvir and sofosbuvir administration in patients with HCV genotype 1 and HIV co-infection. JAMA. 2015;313(12):1232-9. doi: 10.1001/jama.2015.1373. [PubMed: 25706232].

40. Younossi ZM, Stepanova M, Sulkowski M, Naggie S, Henry L, Hunt S. Sofosbuvir and ledipasvir improve patient-reported outcomes in patients co-infected with hepatitis $C$ and human immunodeficiency virus. J Viral Hepat. 2016;23(11):857-65. doi: 10.1111/jvh.12554. [PubMed: 27291391].

41. Ingiliz P, Christensen S, Kimhofer T, Hueppe D, Lutz T, Schewe K, et al. Sofosbuvir and ledipasvir for 8 weeks for the treatment of chronic hepatitis $\mathrm{C}$ virus (HCV) infection in HCV-monoinfected and HIV-HCVcoinfected individuals: Results from the German hepatitis $\mathrm{C}$ cohort (GECCO-01). Clin Infect Dis. 2016;63(10):1320-4. doi: 10.1093/cid/ciw567. [PubMed: 27535952].

42. Steiner S, Bucsics T, Schwabl P, Mandorfer M, Scheiner B, Aichelburg MC, et al. Progress in eradication of HCV in HIV positive patients with significant liver fibrosis in Vienna. Wien Klin Wochenschr. 2017;129(15-16):517-26. doi: 10.1007/s00508-016-1162-y. [PubMed:
28130599]. [PubMed Central: PMC5552846].

43. Bhattacharya D, Belperio PS, Shahoumian TA, Loomis TP, Goetz MB, Mole LA, et al. Effectiveness of all-oral antiviral regimens in $996 \mathrm{hu}-$ man immunodeficiency virus/hepatitis $C$ virus genotype 1-coinfected patients treated in routine practice. Clin Infect Dis. 2017;64(12):1711-20. doi: 10.1093/cid/cix111. [PubMed: 28199525].

44. Luetkemeyer AF, McDonald C, Ramgopal M, Noviello S, Bhore R, Ackerman P. 12 weeks of daclatasvir in combination with sofosbuvir for HIV-HCV coinfection (ALLY-2 study): Efficacy and safety by HIV combination antiretroviral regimens. Clin Infect Dis. 2016;62(12):148996. doi: 10.1093/cid/ciw163. [PubMed: 27025835]. [PubMed Central: PMC4885650].

45. Mandorfer M, Schwabl P, Steiner S, Chromy D, Bucsics T, Stättermayer AF, et al. Interferon-free treatment with sofosbuvir/daclatasvir achieves sustained virologic response in $100 \%$ of HIV/HCV-coinfected patients with advanced liver disease. Z Gastroenterol. 2016;54(5). doi: 10.1055/s-0036-1584038.

46. Wyles DL, Ruane PJ, Sulkowski MS, Dieterich D, Luetkemeyer A, Morgan TR, et al. Daclatasvir plus sofosbuvir for HCV in patients coinfected with HIV-1. N Engl J Med. 2015;373(8):714-25. doi: 10.1056/NEJMoa1503153. [PubMed: 26196502].

47. Castells L, Llaneras J, Campos-Varela I, Bilbao I, Crespo M, Len O, et al. Sofosbuvir and daclatasvir in mono- and HIV-coinfected patients with recurrent hepatitis C after liver transplant. Ann Hepatol. 2017;16(1):8693. doi: 10.5604/16652681.1226819. [PubMed: 28051797].

48. Rockstroh JK, Ingiliz P, Petersen J, Peck-Radosavljevic M, Welzel TM, Van der Valk M, et al. Daclatasvir plus sofosbuvir, with or without ribavirin, in real-world patients with HIV-HCV coinfection and advanced liver disease. Antivir Ther. 2017;22(3):225-36. doi: 10.3851/IMP3108. [PubMed: 27845298].

49. Sulkowski M, Hezode C, Gerstoft J, Vierling JM, Mallolas J, Pol S, et al. Efficacy and safety of 8 weeks versus 12 weeks of treatment with grazoprevir (MK-5172) and elbasvir (MK-8742) with or without ribavirin in patients with hepatitis $C$ virus genotype 1 mono-infection and HIV/hepatitis C virus co-infection (C-WORTHY): A randomised, openlabel phase 2 trial. Lancet. 2015;385(9973):1087-97. doi: 10.1016/S01406736(14)61793-1. [PubMed: 25467560].

50. Massimo A, Teti E, Antinori A, Milazzoi L, Sollima S, Rizzardini G, et al. Ombitasvir/Paritaprevir/Ritonavir and Dasabuvir Combination Treatment in Patients with HIV/HCV Co-Infection: Results of an Italian Compassionate Use Program. Clin Infect Dis. 2017;64(5):680-3. doi: 10.1093/cid/ciw846. [PubMed: 28011605].

51. Younossi ZM, Stepanova M, Sulkowski M, Wyles D, Kottilil S, Hunt S. Patient-reported outcomes in patients co-infected with hepatitis $\mathrm{C}$ virus and human immunodeficiency virus treated with sofosbuvir and velpatasvir: The ASTRAL-5 study. Liver Int. 2017;37(12):1796-804. doi: 10.1111/liv.13462. [PubMed: 28470938].

52. Buti M, Calleja JL, Forns X, Diago M, Ortega E, Crespo J, et al. Simeprevir plus sofosbuvir for hepatitis $C$ virus genotype 4 infection: A phase 3, open-label study. J Hepatol. 2016;64(2):S220-1. doi: 10.1016/s01688278(16)00193-8.

53. Pawlotsky JM, Negro F, Aghemo A, Berenguer M, Dalgard O, Dusheiko G, et al. EASL recommendations on treatment of hepatitis C 2018. I Hepatol. 2018;69(2):461-511. doi:10.1016/j.jhep.2018.03.026.

54. European Association for the Study of the Liver; Electronic address. EASL Recommendations on Treatment of Hepatitis C 2016.J Hepatol. 2017;66(1):153-94. doi:10.1016/j.jhep.2016.09.001. [PubMed: 27667367].

55. Desnoyer A, Pospai D, Le MP, Gervais A, Heurgue-Berlot A, Laradi A, et al. Pharmacokinetics, safety and efficacy of a full dose sofosbuvirbased regimen given daily in hemodialysis patients with chronic hepatitis C. J Hepatol. 2016;65(1):40-7. doi: 10.1016/j.jhep.2016.02.044. [PubMed: 26952005].

56. Guaraldi G, Rossotti R, Verucchi G, Tavio M, Pasulo L, Beghetto $B$, et al. Successful pre- and posttransplant sofosbuvir-based antihepatitis $\mathrm{C}$ virus treatment in persons living with human immun- 
odeficiency virus infection. Open Forum Infect Dis. 2017;4(2):ofx065. doi: 10.1093/ofid/ofx065. [PubMed: 28584851]. [PubMed Central: PMC5451199].

57. Rezaee-Zavareh MS, Hesamizadeh K, Behnava B, Alavian SM, GholamiFesharaki M, Sharafi H. Combination of ledipasvir and sofosbuvir for treatment of hepatitis $C$ virus genotype 1 infection: Systematic review and meta-analysis. Ann Hepatol. 2017;16(2):188-97. doi: 10.5604/16652681.1231562. [PubMed: 28233739].

58. Wyles D, Saag M, Viani RM, Lalezari J, Adeyemi O, Bhatti L, et al. TURQUOISE-I part 1b: Ombitasvir/paritaprevir/ritonavir and dasabuvir with ribavirin for hepatitis $C$ virus infection in HIV-1 coinfected patients on darunavir. J Infect Dis. 2017;215(4):599-605. doi: 10.1093/infdis/jiw597. [PubMed: 28329334].

59. Agarwal K, Dumas EO, Gaeta GB, Lee S, Streinu-Cercel A, Schott E, et al. Long-term clinical outcomes in HCV genotype 1-infected patients receiving ombitasvir/paritaprevir/ritonavir and dasabuvir \pm ribavirin: First interim safety and efficacy results from TOPAZ-I. Hepatology. 2016;64:427.

60. Wei L, Hou J, Luo Y, Heo J, Chu CJ, Duan Z, et al. ONYX-I: Safety and efficacy of ombitasvir/paritaprevir/ritonavir and dasabuvir in Asian adults with genotype $1 \mathrm{~b}$ chronic hepatitis $\mathrm{C}$ virus (HCV) infection: A randomized, double-blind, placebo-controlled study. 26th Conference of the Asian Pacific Association for the Study of the Liver(APASL). Feb 15-19; Shanghai, China. 2017.

61. Feld JJ, Moreno C, Trinh R, Tam E, Bourgeois S, Horsmans Y, et al. Sustained virologic response of $100 \%$ in HCV genotype $1 \mathrm{~b}$ patients with cirrhosis receiving ombitasvir/paritaprevir/r and dasabuvir for 12weeks. J Hepatol. 2016;64(2):301-7. doi: 10.1016/j.jhep.2015.10.005. [PubMed: 26476290].

62. Feld JJ, Jacobson IM, Hezode C, Asselah T, Ruane PJ, Gruener N, et al. Sofosbuvir and velpatasvir for HCV genotype 1, 2, 4, 5, and 6 infection. N Engl J Med. 2015;373(27):2599-607. doi: 10.1056/NEJMoa1512610. [PubMed: 26571066].

63. Tsai N, Bacon B, Curry M, Dieterich D, Flamm S, Kowdley K, et al. Utilization of DAA therapies ledipasvir/sofosbuvir and sofosbuvir/velpatasvir in patients with genotype $1 \mathrm{HCV}$ : Real-world experience from the TRIO network. Gastroenterology. 2017;152(5). S1091. doi: 10.1016/S0016-5085(17)33676-4.

64. Wyles D, Brau N, Kottilil S, Daar ES, Ruane P, Workowski K, et al. Sofosbuvir and velpatasvir for the treatment of hepatitis $\mathrm{C}$ Virus in patients coinfected with human immunodeficiency virus type 1: An open-label, phase 3 study. Clin Infect Dis. 2017;65(1):612. doi: 10.1093/cid/cix260. [PubMed: 28369210]. [PubMed Central: PMC6248627].

65. Gower E, Estes C, Blach S, Razavi-Shearer K, Razavi H. Global epidemiol- ogy and genotype distribution of the hepatitis C virus infection. $J$ Hep atol. 2014;61(1 Suppl):S45-57. doi:10.1016/j.jhep.2014.07.027. [PubMed: 25086286].

66. Bryan JP. Viral hepatitis: Update on hepatitis C. Consultant 1995;35(10):1551-4.

67. Kanda T, Imazeki F, Yokosuka O. New antiviral therapies for chronic hepatitis C. Hepatol Int. 2010;4(3):548-61. doi: 10.1007/s12072-010-91933. [PubMed: 21063477]. [PubMed Central: PMC2940000].

68. Hesamizadeh K, Sharafi H, Rezaee-Zavareh MS, Behnava B, Alavian SM. Next steps toward eradication of hepatitis $C$ in the Era of direct acting antivirals. Hepat Mon. 2016;16(4). e37089. doi: 10.5812/hepatmon.37089. [PubMed: 27275164]. [PubMed Central: PMC4893415].

69. Dolatimehr F, Karimi-Sari H, Rezaee-Zavareh MS, Alavian SM, Behnava B, Gholami-Fesharaki M, et al. Combination of sofosbuvir, pegylatedinterferon and ribavirin for treatment of hepatitis $C$ virus genotype 1 infection: A systematic review and meta-analysis. Daru. 2017;25(1):11 doi: 10.1186/s40199-017-0177-x. [PubMed: 28427463]. [PubMed Central: PMC5397824].

70. Afdhal N, Reddy KR, Nelson DR, Lawitz E, Gordon SC, Schiff E, et al. Ledipasvir and sofosbuvir for previously treated HCV genotype 1 infection. N Engl J Med. 2014;370(16):1483-93. doi: 10.1056/NEJMoa1316366. [PubMed: 24725238].

71. Ferenci P, Bernstein D, Lalezari J, Cohen D, Luo Y, Cooper C, et al ABT-450/r-ombitasvir and dasabuvir with or without ribavirin for HCV. N Engl J Med. 2014;370(21):1983-92. doi: 10.1056/NEJMoa1402338. [PubMed: 24795200].

72. Sharafi H, Nikbin M, Alavian SH, Behnava B, Alavian SM. Efficacy and safety of generic sofosbuvir/ledipasvir fixed-dose combination in Iranian patients with chronic hepatitis $\mathrm{C}$ virus infection. Hepat Mon. 2017;17(6). e12216. doi: 10.5812/hepatmon.12216.

73. Karageorgopoulos DE, Allen J, Bhagani S. Hepatitis C in human immunodeficiency virus co-infected individuals: Is this still a "special population"? World J Hepatol. 2015;7(15):1936-52. doi: 10.4254/wjh.v7.i15.1936. [PubMed: 26244068]. [PubMed Central: PMC4517153].

74. Department of Health and Human Services. Panel on Clinical Practices for Treatment of HIV Infection; Henry J. Kaiser Family Foundation. Panel on Clinical Practices for Treatment of HIV Infection. Guidelines for the use of antiretroviral agents in HIV-infected adults and adolescents, January 28, 2000 by the panel on clinical practices for treatment of HIV infection. HIV Clin Trials. 2000;1(1):60-110. doi 10.1310/hct.2000.1.1.008. [PubMed: 11590490].

75. European AIDS Clinical Society. Guidelines version 8.0. October. Brussels, Belgium; 2015. Available from: http://www.eacsociety.org/files/ guidelines-8.0-english.pdf. 
Table 1. Characteristics and Results of the Included Studies (ITT, Intention-to-Treat; PP, Per-Protocol; ND, Non-Determined)

\begin{tabular}{|c|c|c|c|c|c|c|c|}
\hline Regimen/Author & Year & Sample Size & Duration (Week) & HCV Genotype & Ribavirin & SVR Protocol & SVR12, \% \\
\hline \multicolumn{8}{|l|}{ Sofosbuvir plus simeprevir } \\
\hline Del Bello et al. (24) & 2016 & 41 & 12 & 1 & No & ITT & 75.6 \\
\hline Del Bello et al. (24) & 2016 & 17 & 12 & 1 & Yes & ITT & 94.1 \\
\hline Johnson et al. (25) & 2016 & 13 & 12 & 1 & No & ITT & 92.3 \\
\hline Grant et al. (26) & 2016 & 6 & 12 & 1 & No & PP & 100 \\
\hline Hawkins et al. (27) & 2016 & 33 & 12 & ND & No & PP & 96.9 \\
\hline Merli et al. (28) & 2016 & 12 & 12 & $1 \mathrm{a}, 1 \mathrm{~b}, 4$ & Mix & ITT & 100 \\
\hline Milazzo et al. (29) & 2016 & 11 & ND & $1 \mathrm{a}, 1 \mathrm{~b}, 4$ & Mix & ITT & 72.7 \\
\hline Patel et al. (13) & 2016 & 17 & 12 & ND & No & ITT & 100 \\
\hline Bruno et al. (30) & 2017 & 29 & 12 & $1 \mathrm{a}, 1 \mathrm{~b}, 4$ & Mix & ITT & 86.2 \\
\hline Montes et al. (31) & 2017 & 23 & ND & 1a, 1b, 4, Mix/Other & Mix & ITT & 82.6 \\
\hline Piroth et al. (32) & 2016 & 19 & 12,24 & 1,4 & Mix & ITT & 89.4 \\
\hline \multicolumn{8}{|l|}{ Sofosbuvir plus ribavirin } \\
\hline Del Bello et al. (24) & 2016 & 31 & 12 & 1 & Yes & ITT & 51.6 \\
\hline Campos-Varela et al. (33) & 2016 & 10 & 24 & ND & Yes & $\mathrm{PP}$ & 80 \\
\hline Grant et al. (26) & 2016 & 7 & 12 & ND & Yes & $\mathrm{PP}$ & 57.1 \\
\hline Molina et al. (34) & 2013 & 274 & 24 & $1,2,3,4$ & Yes & ITT & 86.4 \\
\hline Younossi et al. (35) & 2015 & 497 & 12,24 & $1,2,3,4$ & Yes & ITT & 83 \\
\hline Sulkowski et al. (36) & 2014 & 223 & 12,24 & $1 a, 1 b, 2,3$ & Yes & ITT & 78.9 \\
\hline El Sayed et al. (37) & 2017 & 12 & 12 & $1 a, 1 b$ & Yes & ITT & 91.6 \\
\hline Patel et al. (13) & 2016 & 7 & 12 & ND & Yes & ITT & 85.7 \\
\hline Piroth et al. (32) & 2016 & 51 & 12,24 & 2,3 & Yes & ITT & 86.2 \\
\hline \multicolumn{8}{|l|}{ Sofosbuvir/ledipasvir } \\
\hline Johnson et al. (25) & 2016 & 15 & 12 & 1 & No & ITT & 100 \\
\hline Cooper et al. (38) & 2016 & 9 & 24 & $1 \mathrm{a}, 1 \mathrm{~b}$ & Yes & ITT & 88.8 \\
\hline Grant et al. (26) & 2016 & 11 & 12 & ND & No & PP & 100 \\
\hline Naggie et al. (16) & 2015 & 335 & 12 & $1 \mathrm{a}, 1 \mathrm{~b}, 4$ & No & ITT & 96.1 \\
\hline Osinusi et al. (39) & 2015 & 50 & 12 & $1 \mathrm{a}, 1 \mathrm{~b}$ & No & ITT & 98 \\
\hline Younossi et al. (40) & 2016 & 335 & 12 & 1,4 & No & ITT & 96.1 \\
\hline Ingiliz et al. (41) & 2016 & 28 & 8 & 1,4 & No & PP & 96.4 \\
\hline Steiner et al. (42) & 2016 & 19 & 12,24 & 1,3 & No & $\mathrm{PP}$ & 100 \\
\hline Bhattacharya et al. (43) & 2017 & 119 & 12 & 1 & Yes & $\mathrm{PP}$ & 90.7 \\
\hline Bhattacharya et al. (43) & 2017 & 569 & 12 & 1 & No & $\mathrm{PP}$ & 95.2 \\
\hline Patel et al. (13) & 2016 & 137 & 12 & ND & No & ITT & 98.5 \\
\hline Montes et al. (31) & 2017 & 288 & ND & 1a, 1b, 4, Mix/Other & Mix & ITT & 93.7 \\
\hline Piroth et al. (32) & 2016 & 56 & 12,24 & $1,4,6$ & No & ITT & 96.4 \\
\hline Piroth et al. (32) & 2016 & 10 & 12,24 & $1,4,6$ & Yes & ITT & 96.2 \\
\hline \multicolumn{8}{|l|}{ Sofosbuvir plus daclatasvir } \\
\hline Luetkemeyer et al. (44) & 2016 & 151 & 12 & $1 \mathrm{a}, 1 \mathrm{~b}, 2,3,4$ & No & PP & 97.3 \\
\hline
\end{tabular}




\begin{tabular}{|c|c|c|c|c|c|c|c|}
\hline Mandorfer et al. (45) & 2016 & 31 & 12,24 & $1,3,4$ & No & ITT & 100 \\
\hline Wyles et al. (46) & 2015 & 203 & 8,12 & $1,2,3,4$ & No & ITT & 92.1 \\
\hline Castells et al. (47) & 2017 & 6 & 24 & $1 a, 1 b, 2,3$ & No & ITT & 100 \\
\hline Milazzo et al. (29) & 2016 & 18 & ND & $1 a, 1 b, 3,4$ & Mix & ITT & 100 \\
\hline Rockstroh et al. (48) & 2017 & 39 & 24 & $1 \mathrm{a}, 1 \mathrm{~b}, 3,4, \mathrm{Mix}$ & No & ITT & 84.6 \\
\hline Rockstroh et al. (48) & 2017 & 16 & 24 & 1a, 1b, 3, Mix, Unknown & Yes & ITT & 93.7 \\
\hline Montes et al. (31) & 2017 & 74 & ND & 1a, 1b, 3, 4, Mix/Other & Mix & ITT & 93.2 \\
\hline Piroth et al. (32) & 2016 & 156 & 12,24 & $1,2,3,4,6$ & No & ITT & 95.5 \\
\hline Piroth et al. (32) & 2016 & 25 & 12,24 & $1,2,3,4,6$ & Yes & ITT & 92 \\
\hline \multicolumn{8}{|l|}{ Grazoprevir/Elbasvir } \\
\hline Rockstroh et al. (17) & 2015 & 218 & 12 & $1 a, 1 b, 4,6$ & No & ITT & 96.3 \\
\hline Sulkowski et al. (49) & 2015 & 30 & 12 & $1 \mathrm{a}, 1 \mathrm{~b}$ & No & ITT & 86.6 \\
\hline Sulkowski et al. (49) & 2015 & 29 & 12 & $1 \mathrm{a}, 1 \mathrm{~b}$ & Yes & ITT & 96.5 \\
\hline \multicolumn{8}{|c|}{$\begin{array}{l}\text { Ombitasvir/paritaprevir/ritonavir plus } \\
\text { dasabuvir }\end{array}$} \\
\hline Sulkowski et al. (2) & 2015 & 31 & 12 & 1 & Yes & ITT & 93.5 \\
\hline Sulkowski et al. (2) & 2015 & 32 & 24 & 1 & Yes & ITT & 90.6 \\
\hline Bhattacharya et al. (43) & 2017 & 55 & 12 & 1 & Yes & $\mathrm{PP}$ & 90.9 \\
\hline Bhattacharya et al. (43) & 2017 & 23 & 12 & 1 & No & PP & 95.6 \\
\hline Milazzo et al. (29) & 2016 & 22 & ND & $1 \mathrm{a}, 1 \mathrm{~b}$ & Mix & ITT & 90.9 \\
\hline Wyles et al. (58) & 2017 & 22 & 12 & 1 & Yes & ITT & 100 \\
\hline Montes et al. (31) & 2017 & 88 & ND & 1a, $1 \mathrm{~b}, \mathrm{Mix} /$ Other & Mix & ITT & 98.8 \\
\hline Massimo et al. (50) & 2016 & 210 & 12,24 & 1 & Mix & ITT & 96.6 \\
\hline \multicolumn{8}{|l|}{ Sofosbuvir/Velpatasvir } \\
\hline Younossi et al. (51) & 2017 & 106 & 12 & $1,2,3,4$ & No & ITT & 95.2 \\
\hline
\end{tabular}

